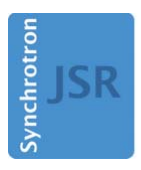

JOURNAL OF SYNCHROTRON RADIATION

ISSN 1600-5775

Received 5 July 2016

Accepted 17 October 2016

Edited by A. Momose, Tohoku University, Japan

Keywords: X-ray; beam metrology; Fresnel diffraction; fourth-generation source; SACLA; LiF; X-ray imaging detector; color centers.

\section{Coherent X-ray beam metrology using 2D high-resolution Fresnel-diffraction analysis}

\author{
M. Ruiz-Lopez, ${ }^{\text {a }}$ A. Faenov, ${ }^{b, c *}$ T. Pikuz, ${ }^{\text {c,d }}$ N. Ozaki, ${ }^{\text {d,e }}$ A. Mitrofanov, ${ }^{c}$ \\ B. Albertazzi, ${ }^{d}$ N. Hartley, ${ }^{d}$ T. Matsuoka, ${ }^{b}$ Y. Ochante, ${ }^{d}$ Y. Tange, ${ }^{f}$ T. Yabuuchi, ${ }^{g}$ \\ T. Habara, ${ }^{\text {d }}$ K. A. Tanaka, ${ }^{\text {d,e }}$ Y. Inubushi, ${ }^{f}$ M. Yabashi, ${ }^{f, g}$ M. Nishikino, ${ }^{\text {h }}$ T. Kawachi, \\ S. Pikuz, ${ }^{c}$ T. Ishikawa, ${ }^{f, g}$ R. Kodama ${ }^{b, d, e}$ and D. Bleiner ${ }^{a *}$
}

${ }^{\mathbf{a} E m p a, ~ M a t e r i a l s ~ S c i e n c e ~ a n d ~ T e c h n o l o g y, ~ D u ̈ b e n d o r f, ~ S w i t z e r l a n d, ~}{ }^{\mathbf{b}}$ Institute for Academic Initiatives, Osaka University, Suita, Osaka, Japan, ' Joint Institute for High Temperatures, Russian Academy of Sciences, Moscow, Russia, ${ }^{\mathbf{d}}$ Graduate School of Engineering, Osaka University, Suita, Osaka, Japan, ${ }^{\mathbf{e}}$ Photon Pioneers Center, Osaka University, Suita, Osaka, Japan, 'fJASRI/SPring-8, Sayo, Hyogo, Japan, ${ }^{\mathbf{g}}$ RIKEN Harima Institute, Sayo, Hyogo, Japan, and ${ }^{\mathbf{h}}$ Kansai Photon Research Institute, National Institutes for Quantum and Radiological Science and Technology, Kizugawa, Kyoto, Japan. *Correspondence e-mail: faenov.anatoly@photon.osaka-u.ac.jp, davide.bleiner@empa.ch

Direct metrology of coherent short-wavelength beamlines is important for obtaining operational beam characteristics at the experimental site. However, since beam-time limitation imposes fast metrology procedures, a multiparametric metrology from as low as a single shot is desirable. Here a twodimensional (2D) procedure based on high-resolution Fresnel diffraction analysis is discussed and applied, which allowed an efficient and detailed beamline characterization at the SACLA XFEL. So far, the potential of Fresnel diffraction for beamline metrology has not been fully exploited because its highfrequency fringes could be only partly resolved with ordinary pixel-limited detectors. Using the high-spatial-frequency imaging capability of an irradiated LiF crystal, 2D information of the coherence degree, beam divergence and beam quality factor $M^{2}$ were retrieved from simple diffraction patterns. The developed beam metrology was validated with a laboratory reference laser, and then successfully applied at a beamline facility, in agreement with the source specifications.

\section{Introduction}

In the last decade, many coherent beamlines, in soft and hard X-ray domains, e.g. SACLA in Japan, LCLS in the USA, FLASH in Germany, FERMI in Italy, etc., have given access to new investigation opportunities in fields such as material processing (Chapman et al., 2006; Milathianaki et al., 2013), biological imaging (Seibert et al., 2011; Chapman et al., 2011), laboratory astrophysics (Savin et al., 2012), high-energydensity plasmas (Inogamov et al., 2011; Beye et al., 2013; Rudek et al., 2012), etc. The success in all the above-mentioned fields depended, strictly, on the characteristics of the beam at the experimental site. At fourth-generation synchrotrons [X-ray free-electron lasers (XFELs)], for instance, all nominal beam parameters, such as the coherence length, collimation, brightness and space or time stability (David et al., 2011; Ishikawa et al., 2012; Tono et al., 2013a; Boutet \& Williams, 2010), are so unique in comparison with any other source for spectrometry and imaging (Bleiner \& Ruiz-Lopez, 2014; Bleiner et al., 2005). For comparison, the spatial coherence length in table-top laser-plasma-driven X-ray lasers (Bleiner $e t$ al., 2011, 2014) is as low as $10 \mu \mathrm{m}$ (Ruiz-Lopez \& Bleiner, 2014), whereas XFELs typically achieve spatial coherence 
lengths of hundreds of micrometers and up to millimeters (Singer et al., 2008; Gutt et al., 2012). However, beamlines are complex machines, where metrology on the pointing stability, spatial coherence and divergence are important in order to verify that the unique specifications are confirmed at the experimental end-station.

Limited beam-time, however, demands fast beam metrology methods. Several fast beamline-metrology methods have been developed to monitor X-ray beams. For instance, the spatial coherence length may be measured with a grating interferometer, using the Talbot effect or other interferometric techniques such as the Moiré effect (Yang et al., 2000). These techniques are single-shot, which allows investigation of the wavefront in each individual pulse (Kayser et al., 2016). With them, one obtains information about the wavefront radius of the synchrotron radiation (Diaz et al., 2010). Others have measured the spatial coherence using ptychographic imaging, which allows simultaneous characterization of aberrations in the X-ray focusing optics (Ditmire et al., 1996).

The most popular metrology, however, is the double-slit technique (Ditmire et al., 1996; Liu et al., 2006; Ruiz-Lopez \& Bleiner, 2014). Indeed, this diagnostic has intrinsic advantages such as: (i) the elimination of complex optical instrumentation and related aberrations, and (ii) rapid in situ readout. The technique consists of illuminating a field stop with two parallel one-dimensional cuts using a short-wavelength beam and observing the diffraction pattern. The double slit produces a Fraunhofer diffraction pattern in the far-field (FF), where a CCD camera can record the data. The main advantage of this technique is that the visibility, or contrast, of the fringes in the diffraction pattern has a straightforward correlation with the spatial coherence of the source. Kohn et al. (2000) proposed an advanced approach, which analyzes the Fresnel diffraction pattern produced in the near-field (NF). This procedure permits obtaining information about the spatial coherence length as well as the source size simultaneously, and has been validated at the Pohang Light Source II synchrotron (Park et al., 2014). The results showed excellent agreement with other state-of-the-art beam metrology techniques.

However, all the methods discussed so far have a common drawback in that they are limited to one parameter per test. Furthermore, it is necessary to rotate the measurement frame in order to obtain information about horizontal and vertical orientations of the full beam cross-section. In fact, it is well known that the coherence, the beam quality, the beam collimation, the pointing stability, etc. of an accelerator-based light source may be different in the horizontal (in-plane acceleration) and vertical directions (Kayser et al., 2016; Tono et al., 2013a). Again, in order to obtain information about the coherence degree, beam divergence, beam quality factor $M^{2}$, pointing stability, etc., several individual measurements have to be made. A full-characterization metrology on a single-shot basis, at the short-wavelength of operation of the advanced beamlines, is thus highly desirable.

The aim of this work was to develop and validate a fullcharacterization metrology for coherent beamlines. This multi-parametric advanced analytical technique was possible thanks to LiF detectors, providing in the NF detailed Fresnel patterns. Results were validated with a reference table-top laser in the visible spectral range, and it was successfully applied to the SACLA XFEL beamline in the hard X-ray regime. The discussed method is accurate, single-shot, realised using ordinary and compact equipment and could be widely used for characterization and optimization of different coherent soft and hard X-ray sources.

It should be stressed that our high-resolution Fresnel diffraction analysis (HR-FDA) using a rectangular field mask (here called 'probe-mask' or simply 'mask') has a twodimensional (2D) metrology advantage that has so far not been accomplished. This is because Fresnel diffraction leads to a fine-structured pattern, which an ordinary low spatial-resolution detector smears out, thereby losing much of the valuable detailed information about the incoming beam. The ideal detector for that is based on LiF crystals or thin films. XUVirradiated $\mathrm{LiF}$ hosts stable color centers, which can be used for imaging purposes (Baldacchini et al., 2005). The principle of operation of a LiF crystal is simple. As a first step the crystal is exposed to the $\mathrm{X}$-ray radiation, which generates color centers. $\mathrm{LiF}$ has an absorption band from 440 to $480 \mathrm{~nm}$. It hosts a photo-emission band (photo-fluorescense) in the visible spectral range, which allows readout of the images using commercial microscopes such as confocal fluorescence microscopes.

The main advantages of $\mathrm{LiF}$ detectors for our application are: (i) LiF can acquire high-spatial-frequency images, with resolution smaller than $1 \mu \mathrm{m}$ (Baldacchini et al., 2005), and (ii) $\mathrm{LiF}$ crystals have been demonstrated to work successfully as an X-ray detector (Heidari Bateni et al., 2013; Bonfigli et al., 2013) for measurements of VUV FEL (Pikuz et al., 2012, 2013) and XFEL beams structures (Pikuz et al., 2015). The combination of $\mathrm{LiF}$ technology with Fresnel diffraction beam metrology is fundamental for capturing the tiniest fringes.

The structure of this paper is as follows. In $\S 2$, we discuss the theoretical background of the HR-FDA. The experimental procedure is explained in $\S 3$. The results of the experimental validation realised with a visible table-top laser are given in \$4.1. A detailed report of the experimental results obtained on the XFEL at SACLA is shown in \$4.2. The results include information on the divergence, pointing stability, coherence length, virtual source size and position, and $M^{2}$ factor. A comparison of the HR-FDA versus other metrologies is given in $\S 5$.

\section{Concept of the HR-FDA beamline metrology}

$\mathrm{X}$-ray diffraction, after illuminating the probe-mask, is described by the Kirchhoff integral as follows:

$$
E(x, y)=\frac{1}{i \lambda z} \iint_{-\infty}^{\infty} \exp (2 i \pi r / \lambda) O(x, y) \mathrm{d} x \mathrm{~d} y,
$$

where $E(x, y)$ is the field amplitude in the image plane, $O(x, y)$ defines the shape and dimensions of the probe-mask object 
(here a simple 2D rectangular window), $z$ is the distance between the object and the detector, $\lambda$ is the illumination wavelength and $r$ is the distance between a point $P$ in the object plane and the corresponding point $P^{\prime}$ in the image plane. Fresnel and Fraunhofer diffraction integrals are approximations of the Kirchhoff integral for the NF and far-field (FF), respectively. The Fresnel approximation is applicable if and only if the following relation is valid:

$$
r \approx 1+\frac{1}{2 z}\left[\left(x_{0}-x\right)^{2}+\left(y_{0}-y\right)^{2}\right]
$$

where $x_{0}$ and $y_{0}$ are the coordinates on the detector plane. Applying the Fresnel approximation to the Kirchhoff's integral, one obtains:

$$
\begin{aligned}
E_{\mathrm{NF}}(x, y)= & \frac{\exp (2 i \pi z / \lambda)}{i \lambda z} \iint_{-\infty}^{\infty} \exp \{(i \pi / \lambda z) \\
& \left.\times\left[\left(x_{0}-x\right)^{2}+\left(y_{0}-y\right)^{2}\right]\right\} O(x, y) \mathrm{d} x \mathrm{~d} y .
\end{aligned}
$$

From (3), since one measures $E_{\mathrm{NF}}(x, y)$, while $O(x, y)$ is known, information about the beam-propagation is retrieved. However, on the detector, it is the intensity $I$, not the field $E$, that is measured. For the specific case of the rectangular mask aperture, such as the one used in HR-FDA metrology, the intensity is defined as follows (Abedin et al., 2007):

$$
\begin{aligned}
I_{\mathrm{NF}}= & \left(I_{0} / 4\right)\left[C\left(\beta_{2}\right)-C\left(\beta_{1}\right)\right]^{2} \\
& +\left[S\left(\beta_{2}\right)-S\left(\beta_{1}\right)\right]^{2}\left[C\left(\beta_{4}\right)-C\left(\beta_{3}\right)\right]^{2} \\
& +\left[S\left(\beta_{4} v\right)-S\left(\beta_{3} v\right) v\right]^{2},
\end{aligned}
$$

where $I_{0}$ is the input intensity before the diffraction and $C(\beta)$ and $S(\beta)$ are operating coefficients of the $\cos$ and $\sin$ components, as follows:

$$
C(\beta)=\int_{0}^{\beta} \cos (\pi a / 2) \mathrm{d} a, \quad S(\beta)=\int_{0}^{\beta} \sin (\pi a / 2) \mathrm{d} a,
$$

where $a$ is the mask dimension. $\beta_{1}, \beta_{2}, \beta_{3}, \beta_{4}$, the limits of the integration, depend on the mask dimensions $\left(a_{x}\right.$ and $\left.a_{y}\right)$ and are defined as

$$
\begin{array}{ll}
\beta_{1}=\sqrt{2 / z \lambda}\left(x+a_{x}\right), & \beta_{2}=\sqrt{2 / z \lambda}\left(x-a_{x}\right), \\
\beta_{3}=\sqrt{2 / z \lambda}\left(x+a_{y}\right), & \beta_{4}=\sqrt{2 / z \lambda}\left(x-a_{y}\right) .
\end{array}
$$

On the other hand, the Fraunhofer approximation, in the FF, requires a larger $z$ distance and can be expressed as $r \approx z$. The Fraunhofer approximation is thus only valid if

$$
r \approx z \gg(\pi / \lambda)\left(x_{0}^{2}+y_{0}^{2}\right)_{\max }
$$

where the subscript 'max' refers to the farthest coordinates with respect to the center. Using the Fraunhofer approximation, one obtains:

$$
E_{\mathrm{FF}}(x, y)=\frac{\exp (2 \pi i z)}{i \lambda^{2} z} \exp (i \pi / \lambda z) \mathrm{FT}[O(x, y)],
$$

which, for the specific case of the rectangular mask aperture, turns into an intensity pattern modeled as follows:

$$
I_{\mathrm{FF}}=I_{0}\left[\frac{\sin \left(\pi a_{x} x / z \lambda\right)}{\pi a_{x} x / z \lambda}\right]^{2}\left[\frac{\sin \left(\pi a_{y} y / z \lambda\right)}{\pi a_{y} y / z \lambda}\right]^{2} .
$$

Compared with the FF pattern, the NF pattern carries a quadratic phase dependence. As observed in Fig. 1(a), Fresnel diffraction produces a pattern that preserves information about the incoming beam wavefront. Then, wavefront information is linearized in the FF (Fraunhofer) diffraction (Fig. 1b). The figure shows the intensity distribution in both the horizontal and vertical direction. The high-frequency component in the Fresnel diffraction pattern is only observed with high spatial-resolution detectors such as the LiF crystal, whose 'pixels' are color centers of atomic size (the effective resolution is, however, limited by the readout technique). Fig. 1 also compares the Fresnel and the Fraunhofer pattern observed under a $\mathrm{LiF}$ crystal (resolution $\sim 0.3 \mu \mathrm{m}$, limited by the readout microscope) and an ordinary $\mathrm{CCD}$ of

a) Fresnel approximation
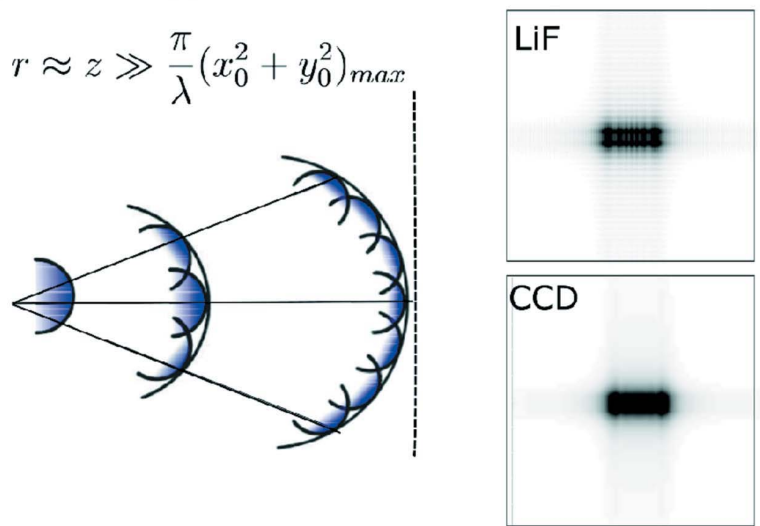

b) Fraunhofer approximation

$$
r \approx 1+\frac{1}{2 z}\left[\left(x_{0}-x\right)^{2}+\left(y_{0}-y\right)^{2}\right]
$$

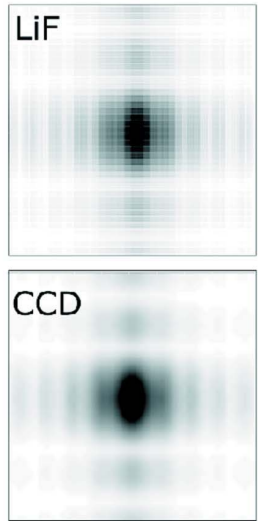

Figure 1

Pattern characteristics as a function of the propagation distance between the diffraction mask and the detector. (a) In Fresnel diffraction each point carries information on the beam wavefront. (b) In Fraunhofer diffraction the signature of the beam characteristics is lost. Both patterns are observed under a $\mathrm{LiF}$ crystal and a CCD. The Fresnel pattern is smeared out if the pattern is detected with the CCD. However, such an effect is not observed in the Fraunhofer diffraction since the higher frequencies are far off the center of the pattern. 

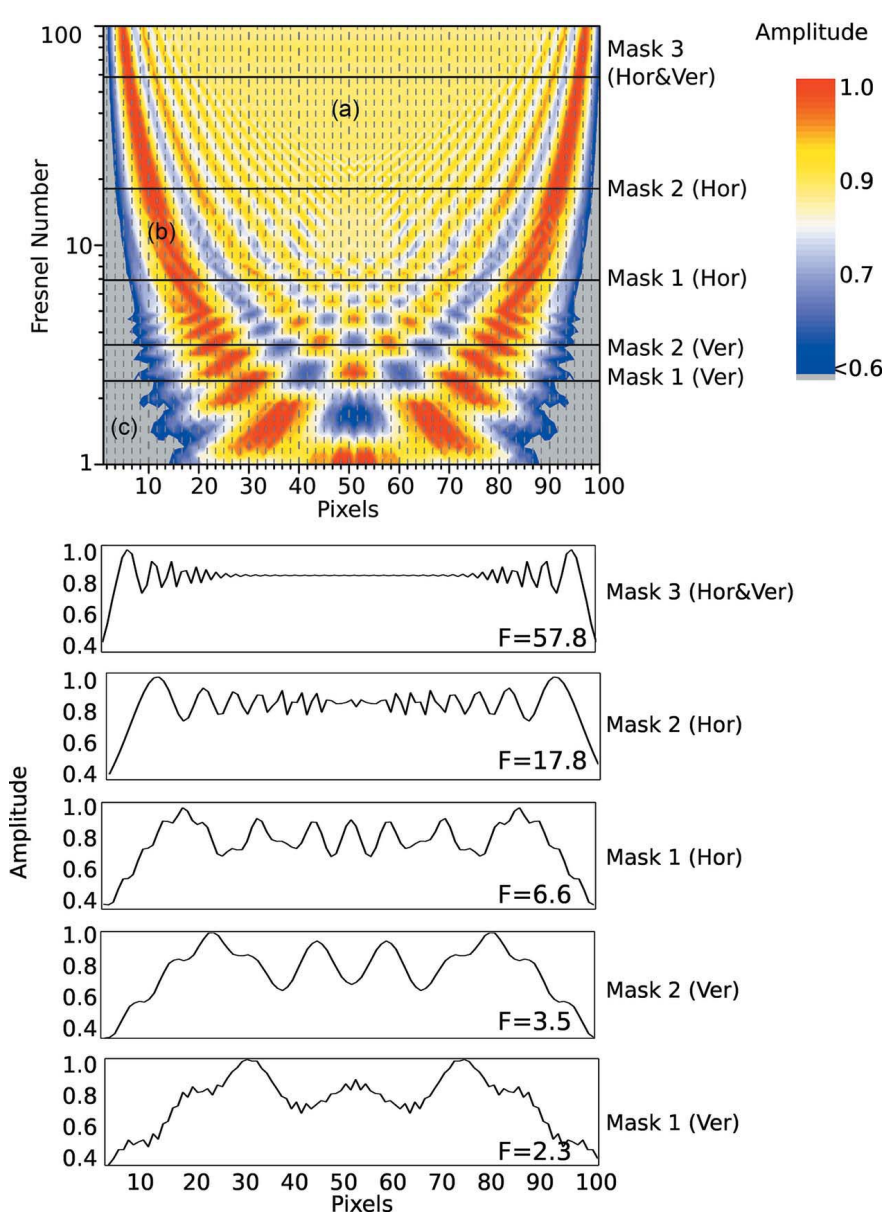

Figure 2

Profile of diffraction patterns of different Fresnel numbers. The color bar indicates the normalized amplitude. The number of peaks (red) at the profile can predict the Fresnel number. The Fresnel numbers of the probe-mask used in this work are marked as Mask $1\left(F_{\mathrm{h}}=6.6, F_{\mathrm{v}}=2.3\right)$, Mask $2\left(F_{\mathrm{h}}=17.8, F_{\mathrm{v}}=3.5\right)$ and Mask $3\left(F_{\mathrm{h}}=F_{\mathrm{v}}=57.8\right)$ and their pattern profiles are observed at the bottom.

$13 \mu \mathrm{m}$ pixel $^{-1}$. The Fresnel pattern is indeed smeared out if the pattern is detected with the CCD. However, such an effect is not relevant in Fraunhofer diffraction.

To define the characteristic observation distance of the Fresnel diffraction pattern, i.e. such that the probe-mask is located in the NF, the Fresnel number $F_{\mathrm{N}}$ must be much greater than unity, namely:

$$
F_{\mathrm{N}}(x, y)=a_{x, y}^{2} / L_{\mathrm{d}} \lambda \gg 1
$$

where $a$ is the size of the probe-mask (with respect to the horizontal or vertical dimension), $L_{\mathrm{d}}$ is the distance between the probe-mask and the detector, and $\lambda$ is the illumination wavelength.
In the NF, the pattern shows internal and external fringes, which carry complete information about the input wavefront. A simple analysis of the fringes highlights the fact that the Fresnel number is the number of peaks in the internal modulation. Fig. 2 shows the structure of the diffraction pattern produced by a 100-pixel mask illuminated with a planar wavefront as a function of the Fresnel number. Calculated profiles, for the probe-masks used in this work, are shown at the bottom of Fig. 2.

The internal modulation, labeled (a) in Fig. 2, is the central region with higher amplitude than the external domains, outside the boundary peaks, labeled $(b)$. Noticeably, for larger Fresnel numbers, typically $F>10$, spacing between the internal fringes is small, and they become indistinguishable with an ordinary detector with pixel-limited resolution. The external modulation, labeled (c), as shown in Fig. 2, demonstrates that the dynamic range of the detector must be large enough for detecting the tiny high-frequency fringes in this domain. From this point of view, the high-resolution detection of the pattern has a key enabling character. The potential of HR-FDA was not accomplished earlier because all ordinary detectors, such as radiochromic plates, CCD, CMOS, etc., suffer from high-frequency suppression (low-pass filtering) (O'Connor et al., 2008). It is crucial to implement a detector with high spatial-frequency capability, like the LiF, to enable the present advanced beamline metrology.

\section{Experimental procedure}

Fig. 3 shows a conceptual sketch of the experimental set-up, along with the main parameters retrieved using HR-FDA as a beamline metrology. Self-amplified stimulated emission (SASE) occurs about the virtual source position $L_{\mathrm{s}}=0$ over a Rayleigh length-scale $L_{\text {Rayleigh }}$. The beam freely propagates along $L_{\mathrm{s}}$, until it meets the probe-mask and diffracts. The diffraction of the probe-mask was monitored at the distance

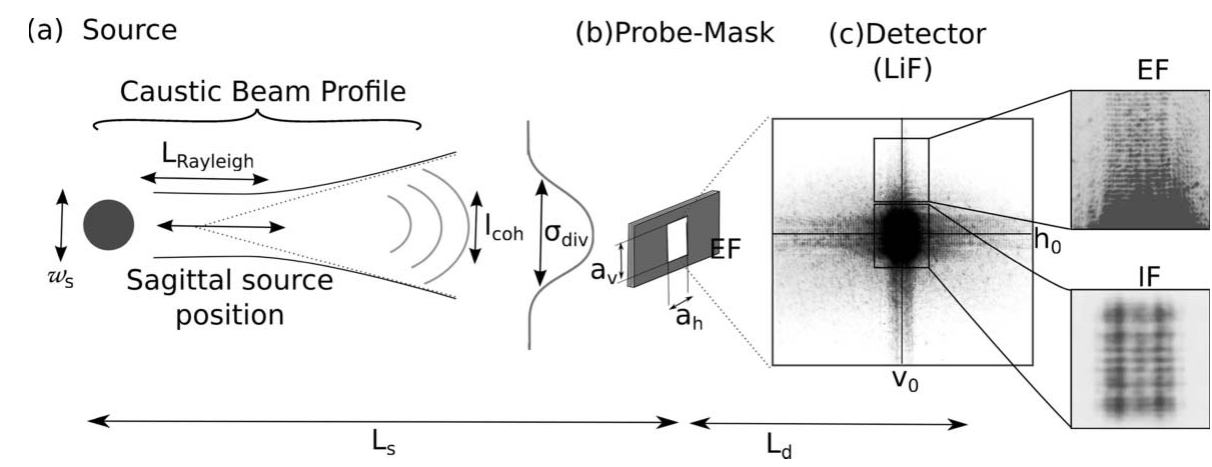

Figure 3

Conceptual sketch of the set-up used for the beam metrology experiments. The X-ray beam propagates from the virtual source position with a caustic profile. The jitter, due to the stochastic generation mechanism of the light, produces pointing instability on the beam. The spatial (transverse) coherence length across the beam is defined by $L_{\text {coh. }}$. When the beam encounters the mask in the near-field, the Fresnel diffraction occurs. The Fresnel pattern is acquired using the $\mathrm{LiF}$ crystal. The pattern is formed by internal (IF) and external (EF) fringe modulations. The beam information is extracted in the near-field from the internal pattern profile. The external pattern is used for a cross-validation. See text for detailed discussion. 
Table 1

Selection of the probe-masks used experimentally and the corresponding Fresnel numbers.

$\mathrm{H}$ : horizontal; $\mathrm{FN}_{\mathrm{h}}$ : horizontal Fresnel number; V: vertical; $\mathrm{FN}_{\mathrm{v}}$ : vertical Fresnel number.

\begin{tabular}{llrrr}
\hline & $\mathrm{H}$ & $\mathrm{FN}_{\mathrm{h}}$ & $\mathrm{V}$ & $\mathrm{FN}_{\mathrm{v}}$ \\
\hline Mask 1 & $164 \mu \mathrm{m}$ & 6.6 & $98 \mu \mathrm{m}$ & 2.3 \\
Mask 2 & $270 \mu \mathrm{m}$ & 17.8 & $120 \mu \mathrm{m}$ & 3.5 \\
Mask 3 & $486 \mu \mathrm{m}$ & 57.8 & $486 \mu \mathrm{m}$ & 57.8 \\
\hline
\end{tabular}

$L_{\mathrm{d}}=8.3 \mathrm{~m}$ from the mask, which was close enough to the detector to produce a Fresnel diffraction pattern (see below). The intensity patterns produced were obtained in situ with a single-shot and acquired with a LiF crystal imaging detector.

Coherent X-ray beam metrology enabled by a Fresnel probe-mask with high spatial-resolution imaging has been carried out at the hard X-ray beamline BL3 of the SACLA facility consisting of: (i) the undulator section, (ii) the electron-beam dump and (iii) the optical hutch and the experimental hall EH5 (Ishikawa et al., 2012). Selection of the probemasks was made as summarized in Table 1. This selection allows comparing the HR-FDA at crucial locations. A photon energy $E_{\mathrm{ph}}=10.1 \mathrm{keV}$ was adopted, well above the LiF cutoff sensitivity of $14 \mathrm{eV}$. At this point, it is important to note that some of the parameters obtained by HR-FDA are photonenergy-dependent and their value may not be reproducible if a different energy is chosen. The pulse energy at the exit of the undulator was $E_{\mathrm{XFEL}}=400 \mu \mathrm{J}\left(2.5 \times 10^{11}\right.$ photons $)$.

The experimental patterns were analyzed with computational algorithms, that would reproduce their shape for a given set of initialization beamline parameters. A self-written iterative code was used to convolute the theoretical patterns and different Gaussian illumination functions, to find the least residuals to the experimental results.

The beamline-of-sight along the optical axis $Z$ fluctuates in both horizontal and vertical directions. The code also allows the centroid position of the statistically averaged fluctuations to be retrieved. Pointing precision is defined in $\mu \mathrm{rad}$ as $1 s_{x, y}$.

The visibility $(\gamma)$ of the fringes, or contrast, is an essential metric to quantify the beam coherence. Visibility is defined as follows:

$$
\gamma=\left|\frac{I_{\max }-I_{\min }}{I_{\max }+I_{\min }}\right|,
$$

where $I_{\max }$ and $I_{\min }$ are, respectively, the maximum and the minimum amplitudes of the fringes at a specific segment of the diffraction pattern. The relationship between the visibility of the fringes and spatial coherence is different in Fraunhofer and Fresnel diffraction. In Fraunhofer diffraction the visibility of the fringes observed in the diffraction pattern has a straightforward correlation with the spatial coherence of the source. In Fresnel diffraction, as shown in Fig. 4, the relation between the visibility and the spatial coherence oscillates between 0 and 1 , for shorter mask lengths, i.e. $<100 \mu \mathrm{m}$. The visibility diminishes for larger mask dimensions, although it remains slightly higher if the spatial coherence length of the

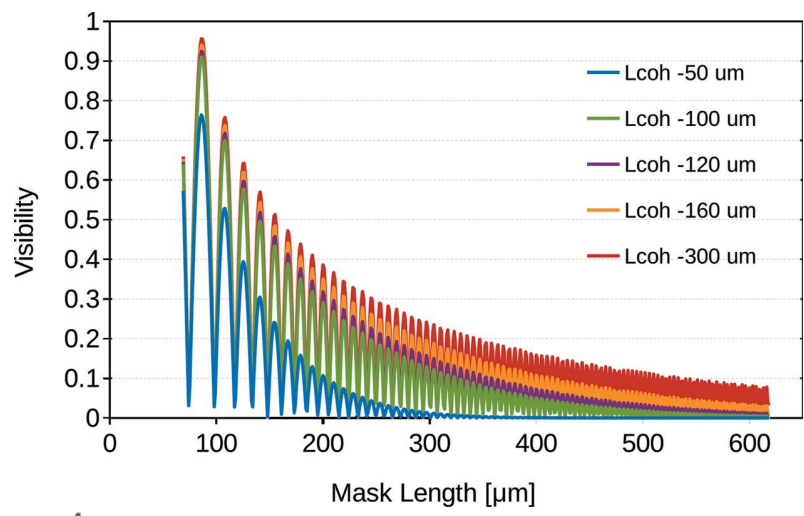

Figure 4

Visibility versus mask length for different coherence lengths. The relation between the visibility and the spatial coherence oscillates between 0 and 1 , for shorter mask lengths. The visibility diminishes for larger mask dimensions although it remains slightly higher if the spatial coherence length of the source is superior.

source is superior. For the present case of Fresnel diffraction the coherence length was calculated using the following equation (Kohn et al., 2000; Park et al., 2014):

$$
l_{\mathrm{coh}}(\gamma)=\frac{a_{x, y}}{\sqrt{8 \ln \left(\gamma_{0} / \gamma\right)}},
$$

where $a_{x, y}$ are the mask size dimensions, $\gamma$ and $\gamma_{0}$ are the visibility of the central fringes for the experimental results and for an ideal point source, defined as follows:

$$
\gamma_{0}=\frac{4 a_{0}}{\pi a}\left|\cos \left[\pi\left(\frac{a_{x, y}^{2}}{a_{0}^{2}}-\frac{3}{4}\right)\right]\right|
$$

where $a_{0}$ is defined as follows:

$$
a_{0}=\left(\frac{4 \lambda L_{\mathrm{d}} L_{\mathrm{s}}}{L_{\mathrm{d}}+L_{\mathrm{s}}}\right)^{1 / 2},
$$

$L_{\mathrm{d}}$ is the distance between the mask and the detector and $L_{\mathrm{s}}$ is the distance between the probe-mask and the source.

\section{Results}

\subsection{Experimental validation with a table-top laser beam}

To begin with, the method was validated in the laboratory using a visible laser source as a standard reference. The beam metrology method was applied to a diode laser (Thorlabs CPS532) whose reference characteristics are available through the manufacturer's data-sheet summarized in Table 2. The

Table 2

Retrieved beam parameters of the visible laser $(\lambda=532 \mathrm{~nm})$ measured using the Fresnel diffraction metrology.

Comparison retrieved parameters and specifications. The accuracy of the measurements is given by $\varepsilon$. $\theta_{\text {div }}$ : divergence angle; $L_{\text {coh }}$; coherence length; $M^{2}$ : beam quality factor.

\begin{tabular}{llll}
\hline Parameter & $\begin{array}{l}\text { This } \\
\text { work }\end{array}$ & $\begin{array}{l}\text { Manufacturer's } \\
\text { specification }\end{array}$ & Accuracy $\varepsilon$ \\
\hline$\theta_{\text {div }}(\mu \mathrm{rad})$ & 0.45 & 0.50 & $-10 \%$ \\
$L_{\text {coh }}(\mu \mathrm{m})$ & 204 & 200 & $2 \%$ \\
$M^{2}$ & 1.7 & 1.9 & $-10 \%$ \\
\hline
\end{tabular}


diffracted patterns were acquired with a $6.5 \mu \mathrm{m} \times 6.5 \mu \mathrm{m}$ pixel-size CMOS. The original beam-width of the diode laser ( $3 \mathrm{~mm}$ ) had to be expanded to illuminate the full 2D mask, increasing its divergence approximately 30-fold. Magnification was corrected during post-processing. The results, summarized in Table 2, demonstrate the accuracy $(\varepsilon)$ of the metrology procedure, given as:

$$
\varepsilon=\frac{x_{\text {meas }}-x_{\text {ref }}}{x_{\text {ref }}} \times 100,
$$

where $x_{\text {meas }}$ and $x_{\text {ref }}$ are the measured and reference values of the diode laser, respectively.

Unfortunately the pixel size of the CMOS was not small enough to resolve the external fringes, or the higher-frequency internal fringes for the horizontal size of the mask, which had a larger Fresnel number $\left(F_{\mathrm{h}}=13.7\right)$. A high-resolution detector such as a LiF crystal would allow more accurate results to be obtained, albeit incompatible at long-wavelength visible radiation wavelength (response cutoff is for $\lambda_{\mathrm{LiF}}<$ $33 \mathrm{~nm})$.

\subsection{Application of the HR-FDA method to the SACLA XFEL}

Fig. 5 shows a diffraction pattern obtained at SACLA XFEL. By processing the mapping in the horizontal and the vertical axis, one obtains the pattern profiles. These profiles were processed with the proprietary data treatment algorithm. The red line in the pattern profile in Fig. 5 corresponds to the 'raw' pattern obtained during the experiments. The blue line corresponds to the pattern calculated for a fully coherent flattop beamline with zero divergence. The black line is the calculated Gaussian illumination with $\sigma=256 \mu \mathrm{m}$ of the Fresnel diffraction pattern giving approximately $75 \%$ coherence degree. From the obtained Fresnel patterns, a number of parameters could be retrieved simultaneously.

4.2.1. Metrology of beam divergence $\left(\theta_{\text {div }}\right)$. The beam divergence was retrieved by means of iterative calculation of Fresnel fringes using a Gaussian illumination, and trying to match the experimental patterns as close as possible. The best fit $\left(R^{2}=0.923\right)$ for the horizontal direction in Mask 1 was considered a converged calculation. The obtained divergence was identical along the horizontal and vertical planes (circular beam cross-section), i.e. $\sigma_{\mathrm{h}}=\sigma_{\mathrm{v}}=256 \mu \mathrm{m}$. The obtained $\sigma_{\mathrm{h}, \mathrm{v}}$ correspond to a Gaussian illumination of $603 \mu \mathrm{m}$ (FWHM). Given the propagation distance $L_{\mathrm{s}}=256 \mathrm{~m}$ one can easily obtain the beam divergence angle. Fig. 6 summarizes the obtained divergences for each experimental probe-mask. The $98 \mu \mathrm{m} \times 164 \mu \mathrm{m}$ probe-mask was acquired twice. The average divergence $\left(\theta_{\text {div }}\right)$ thus obtained is $\theta_{\text {div }}=2.3 \pm 0.4 \mu \mathrm{rad}$ (precision is here given as $1-\sigma$ ).

4.2.2. Metrology of beam pointing $\left(\sigma_{x, y}\right)$. The beamline centroid was retrieved by means of iterative lateral shifting of the Fresnel patterns using a Gaussian illumination, and trying to match the experimental patterns as close as possible. The precision of the beam centroid position was determined to be $\sigma_{x}= \pm 0.3 \mu \mathrm{rad}$ and $\sigma_{y}= \pm 0.2 \mu \mathrm{rad}$ for horizontal and vertical directions, respectively. These values are in agreement with data of Tono et al. (2013b), as summarized on Table 3. The a)
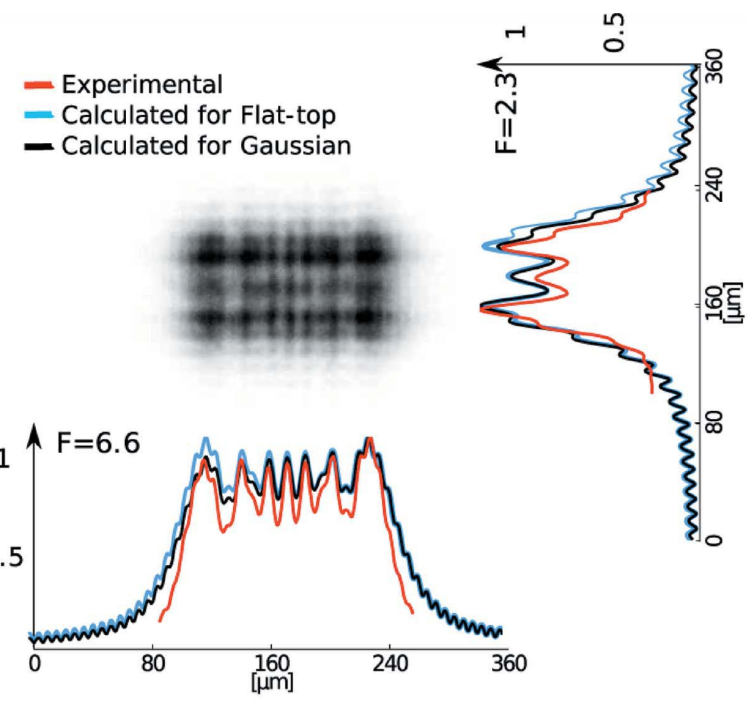

b)
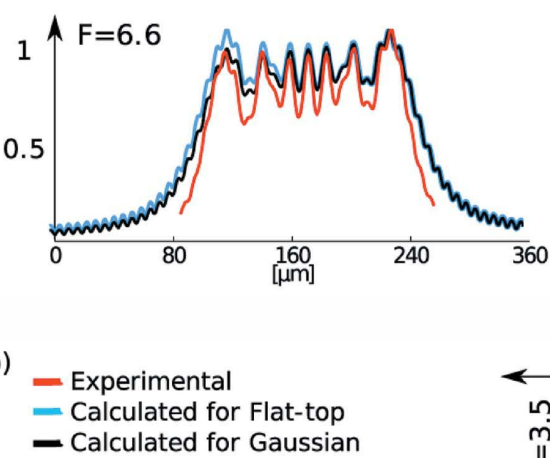

- Calculated for Gaussian

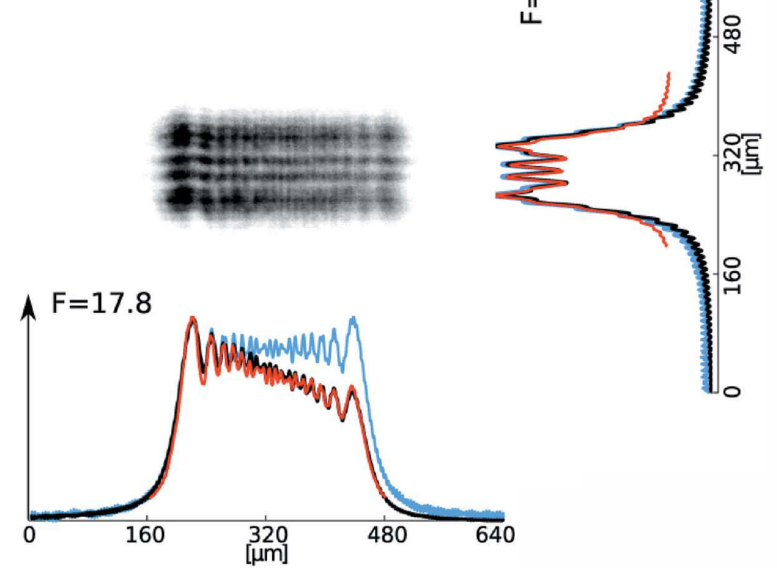

Figure 5

2D experimental diffraction pattern and profiles along the horizontal and vertical directions. Calculated profiles are also shown. The black line is the calculated Gaussian beam $\sigma=256 \mu \mathrm{m}$ of the Fresnel diffraction pattern with approximately $75 \%$ coherence. (a) Mask 1: $164 \mu \mathrm{m} \times 98 \mu \mathrm{m}$, $F_{\mathrm{h}}=6.6, F_{\mathrm{v}}=2.3$. (b) Mask 2: $270 \mu \mathrm{m} \times 120 \mu \mathrm{m}, F_{\mathrm{h}}=17.8, F_{\mathrm{v}}=3.5$.

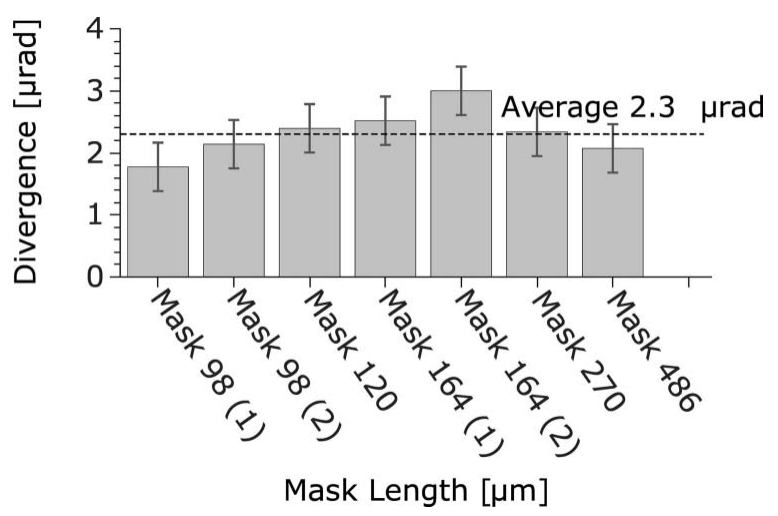

Figure 6

Beam FWHM of the experimental probe-masks. Best match obtained between the divergence of the experimental patterns and the computational patterns. The average is $\theta_{\text {div }}=2.3 \mu \mathrm{rad}$ and the standard deviation is $\pm 0.4 \mu \mathrm{rad}$. 
Table 3

Comparison of results obtained using the present beam metrology and results obtained by others using different metrologies.

$\theta_{\text {div }}:$ divergence; PSH: pointing stability horizontally; PSV: pointing stability vertically; $L_{\text {coh }}$ : coherence length; $M^{2}$ beam quality.

\begin{tabular}{lllc}
\hline Parameter & This work & Others & Accuracy $\varepsilon$ \\
\hline$\theta_{\text {div }}(\mu \mathrm{rad})$ & 2.3 & 2.4 (Ishikawa et al., 2012$)$ & $-4.3 \%$ \\
PSH $(\mu \mathrm{m})$ & $\pm 0.3 \mathrm{H}$ & $\pm 0.4 \mathrm{H}$ (Tono et al., 2013b) & $-33.3 \%$ \\
PSV $(\mu \mathrm{m})$ & $\pm 0.2 \mathrm{H}$ & $\pm 0.2 \mathrm{~V}$ (Tono et al., 2013b) & $0 \%$ \\
$L_{\text {coh }}(\%)$ & 75 & 79 (Lehmkühler et al., 2014) & $-5.3 \%$ \\
$M^{2}$ & 1.65 & 1.6 (Lehmkühler et al., 2014$)$ & $3.1 \%$ \\
\hline
\end{tabular}

different values obtained for the horizontal and vertical directions are thus significant.

4.2.3. Metrology of transverse coherence length $\left(I_{\mathrm{coh}}\right)$. The experimental spatial coherence was retrieved using equation (10), for a given probe-mask size, using the theoretical and experimental visibility. The former is given in equation (11), while the latter is measured. Fig. 7 gives the obtained values as a function of probe-mask size. The transversal coherence scales linearly with the continuous increase of the probe-mask size. The experimental values are in agreement with the theoretical Gaussian values. Table 3 shows that the obtained value of $l_{\text {coh }}=129 \mu \mathrm{m}$ is available for the first time for such a large slit-width. As shown in Fig. 7, a flat-top beam passing through the same probe-mask shows a coherence length only approximately 1.33 times larger than the experimentally obtained one, which confirms a very high degree of SACLA XFEL beam spatial coherence.

4.2.4. Metrology of virtual source size $\left(w_{\mathrm{s}}\right)$. The virtual source radius, i.e. the retro-propagated beam waist of the coherent beam caustic, was determined as follows:

$$
w_{\mathrm{s}}=\frac{\sqrt{2}}{2 \pi} \frac{\lambda L_{\mathrm{s}}}{l_{\mathrm{coh}}}=55 \text { micrometers }
$$

where $L_{\mathrm{s}}$ is the distance between the source and the metrology mask and $l_{\text {coh }}$ is the spatial coherence length. Equation (14) was derived from the analytical expression reported by Kohn

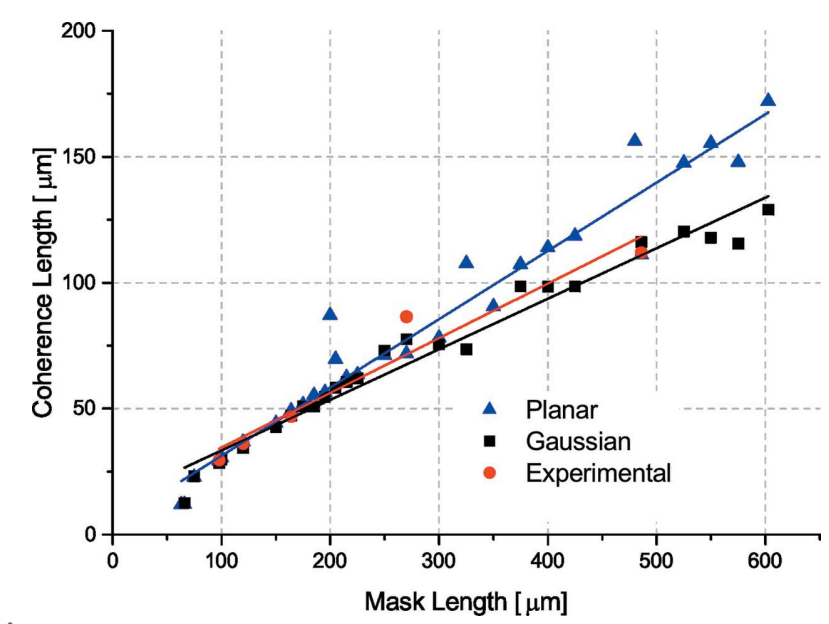

Figure 7

Coherence length as a function of the mask size. Linear regression is shown for the planar, Gaussian and experimental cases.

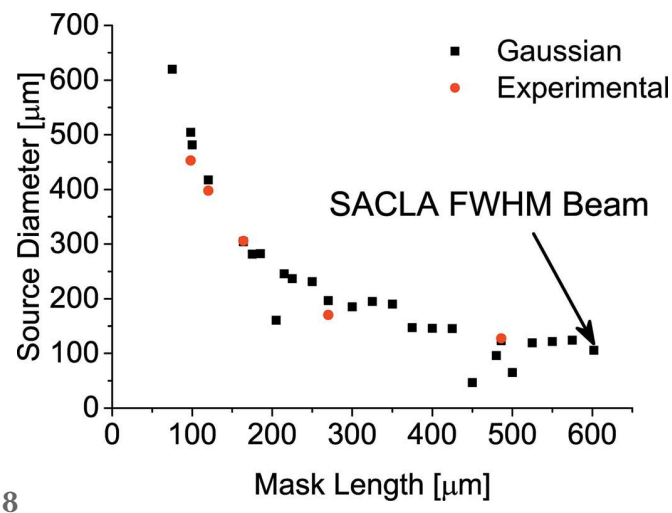

Figure 8

Retrieved virtual source diameter as a function of the mask length. The red circles correspond to the experimental results. The black squares correspond to the calculated Gaussian wavefront. The values settle where the SACLA XFEL value is indicated.

et al. (2000) and Park et al. (2014). Fig. 8 shows an excellent match between the computed Gaussian beam and the experimental one for the virtual source size. Both curves fall exponentially until a probe-mask of approximately $500 \mu \mathrm{m}$. At this point, the value of the virtual source converges and remains consistent around the same number.

4.2.5. Metrology of beam quality factor $\left(M^{2}\right)$. The beam quality factor, $M^{2}$, is defined as the ratio of the beam parameter product of a given beam and an ideal Gaussian beam, where the beam parameter product is the product of the beam radius, measured at the beam waist radius $\left(w_{\mathrm{s}}=55 \mu \mathrm{m}\right)$ and the half beam divergence $\left(\theta_{\text {div }} / 2=1.16 \mu \mathrm{rad}\right) . M^{2}$ is used as the parameter to quantify the beam quality.

For a single-mode $\mathrm{TEM}_{00}$ Gaussian laser beam, $M^{2}$ is equal to unity. This parameter is defined as follows:

$$
M^{2}=\frac{\pi w_{\mathrm{s}} \theta_{\mathrm{div}}}{2 \lambda}=1.65
$$

Therefore, $M^{2}$ in SACLA XFEL is determined to be equal to $M^{2}=1.65$, which is in very good agreement with the designed value.

4.2.6. Metrology of sagittal jitter of the virtual source $\left(\sigma_{z}\right)$. Quantitative analysis of the data shows that the obtained diffraction patterns and the experiments match more closely when a magnification factor is applied. During the data analysis, it was observed that each acquisition of the diffraction patterns is taken with a slightly different magnification. This was due to the sagittal (longitudinal) translation of the source's lasing. For a given fixed imaging plane (the location of the $\mathrm{LiF}$ ) and a given fixed position of the diffraction plane (the location of the mask) the pattern can fluctuate in a self-similar scaling.

Applying a back-propagation procedure, it was possible to retrieve the exact location of the spot source at $-260 \pm 25 \mathrm{~m}$ (after the mask), and assuming a caustic profile of the beam (Fig. 3), the Rayleigh length is calculated for the beam radius $\left(w_{\mathrm{s}}=55 \mu \mathrm{m}\right)$ as follows:

$$
L_{\text {Rayleigh }}=\frac{\pi w_{\mathrm{s}}^{2}}{M^{2} \lambda}=48 \mathrm{~m} .
$$


This indicates that the collimated Rayleigh length covers approximately $95 \%$ of the sagittal jitter $\sigma_{z}$.

\section{Conclusions}

The presented short-wavelength coherent beam metrology method HR-FDA, i.e. based on Fresnel diffraction and a LiF detector, provided single-shot quantitative measurement of the full XFEL parameters. Two important aspects of the LiF detector used in this study were essential in the measurement: resolution and high dynamic range. The computations showed that the width of the last external fringes is approximately $0.3 \mu \mathrm{m}$. Ordinary X-ray cameras with $10-30 \mu \mathrm{m}$ pixel-size would not preserve them. Using $\mathrm{LiF}$ as a detector, the information coming from the higher orders (central part of the field mask), as well as first-orders (edges of the mask), was retrieved. This allowed a full characterization of the SACLA free-electron laser source by retrieving the information in the Fresnel pattern.

The high dynamic range of $\mathrm{LiF}$ permitted measuring the internal and external fringes of the Fresnel diffraction pattern. While the internal ones were used to measure the visibility and thus fully characterize the beam, the external ones were used to make an exhaustive verification of the calculated pattern.

Due to the wide range of sensitivity of the LiF, such detectors can be used for any coherent X-ray beam characterization. However, it is important to note that the characterization of the source depends on the operation condition, i.e. the coherence length depends on the wavelength and the spectral bandwidth of the laser beam.

Table 3 compares results obtained using this beam metrology at SACLA XFEL with different metrologies used by other authors. Previous works have shown that the source was designed to have a divergence of $2.4 \mu \mathrm{rad}$ (Ishikawa et al., 2012; Tono et al., 2013a). HR-FDA results are in agreement with that, having obtained $\theta_{\text {div }}=2.3 \pm 0.4 \mu \mathrm{rad}$. The HR-FDA results in the pointing precision were $0.3 \mu \mathrm{rad}$ in the horizontal axis and $0.2 \mu \mathrm{rad}$ in the vertical axis. The measurements confirmed previous data from the X-ray beamline BL3 (Tono et al., 2013b), where the workers had obtained values for the pointing precision of $0.4 \mu \mathrm{rad}$ (horizontal) and $0.2 \mu \mathrm{rad}$ (vertical). This agrees with our HR-FDA measurements with a deviation of approximately $30 \%$.

The HR-FDA coherence length data indicate a high degree of spatial coherence in agreement with previous prediction (Singer et al., 2008; Vartanyants et al., 2011). The ratio between the visibility of a point source and that of the SACLA XFEL is obtained as high as $75 \%$, where $100 \%$ would indicate a totally planar wavefront. A similar value (79\%) has been found by Lehmkuhler et al. (2014) by analyzing small-angle X-ray scattering speckle patterns from colloidal dispersions. Other FELs have been reported to have a coherence degree of $75 \%$ (Singer et al., 2012). The Rayleigh length has been calculated as $48 \mathrm{~m}$. The evaluation of the X-ray beam realised in SACLA by Kayser et al. (2016) demonstrated a measured Rayleigh length shorter than $80 \mathrm{~m}$. The obtained value of the beam quality parameter $M^{2}$ in SACLA was 1.65. A similar value (1.6) was found by Lehmkühler et al. (2014).

\section{Acknowledgements}

MR-L acknowledges support by the Swiss National Science Foundation under Grant No. PP00P2-133564/1. The XFEL experiments were performed at BL3 of SACLA with the approval of the Japan Synchrotron Radiation Research Institute (JASRI) (Proposals Nos. 2014A8045 and 2014B8068). These experiments were also supported by grants from Grants-in-Aid for Scientific Research (Kakenhi Grant Nos. 22224012, 25289244 and 15H02153) and the Core-toCore Program on International Alliance for Material Science in Extreme States with High Power Laser of the Japan Society for the Promotion of Science (JSPS), from the X-ray FreeElectron Laser Priority Strategy Program of the Ministry of Education, Culture, Sports, Science and Technology (MEXT, contract 12005014). Support by the Russian Foundation for Basics Research (grant 14-22-02089) and by the RAS Presidium Program for basic research (nr11) is also acknowledged.

\section{References}

Abedin, K., Islam, M. \& Haider, A. (2007). Opt. Laser Technol. 39, 237-246.

Baldacchini, G., Bollanti, S., Bonfigli, F., Flora, F., Di Lazzaro, P., Lai, A., Marolo, T., Montereali, R., Murra, D., Faenov, A., Pikuz, T., Nichelatti, E., Tomassetti, G., Reale, A., Reale, L., Ritucci, A., Limongi, T., Palladino, L., Francucci, M., Martellucci, S. \& Petrocelli, G. (2005). Rev. Sci. Instrum. 76, 113104.

Beye, M., Schreck, S., Sorgenfrei, F., Trabant, C., Pontius, N., Schüßler-Langeheine, C., Wurth, W. \& Föhlisch, A. (2013). Nature (London), 501, 191-194.

Bleiner, D., Arbelo-Pena, Y., Masoudnia, L. \& Ruiz-Lopez, M. (2014). Phys. Scr. Vol. T, 162, 4050.

Bleiner, D., Lienemann, P. \& Vonmont, H. (2005). Talanta, 65, 12861294.

Bleiner, D. \& Ruiz-Lopez, M. (2014). Short Wavelength Laboratory Sources, p. 335. Royal Society of Chemistry.

Bleiner, D., Staub, F. \& Balmer, J. E. (2011). Proc. SPIE, 8140, 814014.

Bonfigli, F., Cecilia, A., Bateni, S. H., Nichelatti, E., Pelliccia, D., Somma, F., Vagovic, P., Vincenti, M., Baumbach, T. \& Montereali, R. (2013). Radiat. Meas. 56, 277-280.

Boutet, S. \& Williams, G. J. (2010). New J. Phys. 12, 035024.

Chapman, H. N., Barty, A., Bogan, M. J., Boutet, S., Frank, M., HauRiege, S. P., Marchesini, S., Woods, B. W., Bajt, S., Benner, W. H., London, R. A., Plönjes, E., Kuhlmann, M., Treusch, R., Düsterer, S., Tschentscher, T., Schneider, J. R., Spiller, E., Möller, T., Bostedt, C., Hoener, M., Shapiro, D. A., Hodgson, K. O., van der Spoel, D., Burmeister, F., Bergh, M., Caleman, C., Huldt, G., Seibert, M. M., Maia, F. R. N. C., Lee, R. W., Szöke, A., Timneanu, N. \& Hajdu, J. (2006). Nat. Phys. 2, 839-843.

Chapman, H. N., Fromme, P., Barty, A., White, T. A., Kirian, R. A., Aquila, A., Hunter, M. S., Schulz, J., DePonte, D. P., Weierstall, U., Doak, R. B., Maia, F. R. N. C., Martin, A. V., Schlichting, I., Lomb, L., Coppola, N., Shoeman, R. L., Epp, S. W., Hartmann, R., Rolles, D., Rudenko, A., Foucar, L., Kimmel, N., Weidenspointner, G., Holl, P., Liang, M., Barthelmess, M., Caleman, C., Boutet, S., Bogan, M. J., Krzywinski, J., Bostedt, C., Bajt, S., Gumprecht, L., Rudek, B., Erk, B., Schmidt, C., Hömke, A., Reich, C., Pietschner, D., Strüder, L., Hauser, G., Gorke, H., Ullrich, J., Herrmann, S., Schaller, G., Schopper, F., Soltau, H., Kühnel, K., Messerschmidt, M., Bozek, J. D., Hau-Riege, S. P., Frank, M., Hampton, C. Y., 
Sierra, R. G., Starodub, D., Williams, G. J., Hajdu, J., Timneanu, N., Seibert, M. M., Andreasson, J., Rocker, A., Jönsson, O., Svenda, M., Stern, S., Nass, K., Andritschke, R., Schröter, C., Krasniqi, F., Bott, M., Schmidt, K. E., Wang, X., Grotjohann, I., Holton, J. M., Barends, T. R. M., Neutze, R., Marchesini, S., Fromme, R., Schorb, S., Rupp, D., Adolph, M., Gorkhover, T., Andersson, I., Hirsemann, H., Potdevin, G., Graafsma, H., Nilsson, B. \& Spence, J. C. H. (2011). Nature (London), 470, 73-77.

David, C., Gorelick, S., Rutishauser, S., Krzywinski, J., VilaComamala, J., Guzenko, V. A., Bunk, O., Färm, E., Ritala, M., Cammarata, M., Fritz, D. M., Barrett, R., Samoylova, L., Grünert, J. \& Sinn, H. (2011). Sci. Rep. 1, 57.

Diaz, A., Mocuta, C., Stangl, J., Keplinger, M., Weitkamp, T., Pfeiffer, F., David, C., Metzger, T. H. \& Bauer, G. (2010). J. Synchrotron Rad. 17, 299-307.

Ditmire, T., Gumbrell, E., Smith, R., Tisch, J., Meyerhofer, D. \& Hutchinson, M. (1996). Phys. Rev. Lett. 77, 4756-4759.

Gutt, C., Wochner, P., Fischer, B., Conrad, H., Castro-Colin, M., Lee, S., Lehmkühler, F., Steinke, I., Sprung, M., Roseker, W., Zhu, D., Lemke, H., Bogle, S., Fuoss, P. H., Stephenson, G. B., Cammarata, M., Fritz, D. M., Robert, A. \& Grübel, G. (2012). Phys. Rev. Lett. 108, 024801.

Heidari Bateni, S., Bonfigli, F., Cecilia, A., Baumbach, T., Pelliccia, D., Somma, F., Vincenti, M. \& Montereali, R. (2013). Nucl. Instrum. Methods Phys. Res. A, 720, 109-112.

Inogamov, N., Faenov, A. Y., Zhakhovsky, V., Pikuz, T., Skobelev, I. Y., Petrov, Y. V., Khokhlov, V., Shepelev, V., Anisimov, S., Fortov, V., Fukuda, Y., Kando, M., Kawachi, T., Nagasono, M., Ohashi, H., Yabashi, M., Tono, K., Senda, Y., Togashi, T. \& Ishikawa, T. (2011). Contrib. Plasma Phys. 51, 419-426.

Ishikawa, T., Aoyagi, H., Asaka, T., Asano, Y., Azumi, N., Bizen, T., Ego, H., Fukami, K., Fukui, T., Furukawa, Y., et al. (2012). Nat. Photon. 6, 540-544.

Kayser, Y., Rutishauser, S., Katayama, T., Kameshima, T., Ohashi, H., Flechsig, U., Yabashi, M. \& David, C. (2016). Opt. Lett. 41, 733-736.

Kohn, V., Snigireva, I. \& Snigirev, A. (2000). Phys. Rev. Lett. 85, $2745-$ 2748.

Lehmkühler, F., Gutt, C., Fischer, B., Schroer, M. A., Sikorski, M., Song, S., Roseker, W., Glownia, J., Chollet, M., Nelson, S., Tono, K., Katayama, T., Yabashi, M., Ishikawa, T., Robert, A. \& Grübel, G. (2014). Sci. Rep. 4, 5234.

Liu, Y., Wang, Y., Larotonda, M., Luther, B., Rocca, J. \& Attwood, D. (2006). Opt. Express, 14, 12872-12879.

Milathianaki, D., Boutet, S., Williams, G., Higginbotham, A., Ratner, D., Gleason, A., Messerschmidt, M., Seibert, M. M., Swift, D., Hering, P., Robinson, J., White, W. E. \& Wark, J. S. (2013). Science, 342, 220-223.

O'Connor, P., Frank, J., Geary, J., Gilmore, D., Kotov, I., Radeka, V., Takacs, P. \& Tyson, J. (2008). Proc. SPIE, 7021, 702106.

Park, S. Y., Hong, C. K. \& Lim, J. (2014). Rev. Sci. Instrum. 85, 045116.

Pikuz, T., Faenov, A., Fukuda, Y., Kando, M., Bolton, P., Mitrofanov, A., Vinogradov, A., Nagasono, M., Ohashi, H., Yabashi, M., Tono, K., Senba, Y., Togashi, T. \& Ishikawa, T. (2012). Opt. Express, 20, 3424-3433.

Pikuz, T. A., Faenov, A. Y., Fukuda, Y., Kando, M., Bolton, P., Mitrofanov, A., Vinogradov, A. V., Nagasono, M., Ohashi, H.,
Yabashi, M., Tono, K., Senba, Y., Togashi, T. \& Ishikawa, T. (2013). Appl. Opt. 52, 509-515.

Pikuz, T., Faenov, A., Matsuoka, T., Matsuyama, S., Yamauchi, K., Ozaki, N., Albertazzi, B., Inubushi, Y., Yabashi, M., Tono, K., Sato, Y., Yumoto, H., Ohashi, H., Pikuz, S., Grum-Grzhimailo, A. N., Nishikino, M., Kawachi, T., Ishikawa, T. \& Kodama, R. (2015). Sci. Rep. 5, 17713.

Rudek, B. et al. (2012). Nat. Photon. 6, 858-865.

Ruiz-Lopez, M. \& Bleiner, D. (2014). Appl. Phys. B, 115, 311-324.

Savin, D., Brickhouse, N., Cowan, J., Drake, R., Federman, S., Ferland, G., Frank, A., Gudipati, M., Haxton, W., Herbst, E., Profumo, S., Salama, F., Ziurys, L. M. \& Zweibel, E. G. (2012). Rep. Prog. Phys. 75, 036901.

Seibert, M. M., Ekeberg, T., Maia, F. R., Svenda, M., Andreasson, J., Jönsson, O., Odić, D., Iwan, B., Rocker, A., Westphal, D., Hantke, M., DePonte, D. P., Barty, A., Schulz, J., Gumprecht, L., Coppola, N., Aquila, A., Liang, M., White, T. A., Martin, A., Caleman, C., Stern, S., Abergel, C., Seltzer, V., Claverie, J., Bostedt, C., Bozek, J. D., Boutet, S., Miahnahri, A. A., Messerschmidt, M., Krzywinski, J., Williams, G., Hodgson, K. O., Bogan, M. J., Hampton, C. Y., Sierra, R. G., Starodub, D., Andersson, I., Bajt, S., Barthelmess, M., Spence, J. C. H., Fromme, P., Weierstall, U., Kirian, R., Hunter, M., Doak, R. B., Marchesini, S., Hau-Riege, S. P., Frank, M., Shoeman, R. L., Lomb, L., Epp, S. W., Hartmann, R., Rolles, D., Rudenko, A., Schmidt, C., Foucar, L., Kimmel, N., Holl, P., Rudek, B., Erk, B., Hömke, A., Reich, C., Pietschner, D., Weidenspointner, G., Strüder, L., Hauser, G., Gorke, H., Ullrich, J., Schlichting, I., Herrmann, S., Schaller, G., Schopper, F., Soltau, H., Kühnel, K., Andritschke, R., Schröter, C., Krasniqi, F., Bott, M., Schorb, S., Rupp, D., Adolph, M., Gorkhover, T., Hirsemann, H., Potdevin, G., Graafsma, H., Nilsson, B., Chapman, H. N. \& Hajdu, J. (2011). Nature (London), 470, 78-81.

Singer, A., Sorgenfrei, F., Mancuso, A., Gerasimova, N., Yefanov, O., Gulden, J., Gorniak, T., Senkbeil, T., Sakdinawat, A., Liu, Y., Attwood, D., Dziarzhytski, S., Mai, D. D., Treusch, R., Weckert, E., Salditt, T., Rosenhahn, A., Wurth, W. \& Vartanyants, I. A. (2012). Opt. Express, 20, 17480-17495.

Singer, A., Vartanyants, I., Kuhlmann, M., Duesterer, S., Treusch, R. \& Feldhaus, J. (2008). Phys. Rev. Lett. 101, 254801.

Tono, K., Inubushi, Y., Sato, T., Togashi, T., Ohashi, H., Kimura, H., Takahashi, S., Takeshita, K., Tomizawa, H., Goto, S. \& Yabashi, M. (2013a). J. Phys. Conf. Ser. 425, 072006.

Tono, K., Togashi, T., Inubushi, Y., Sato, T., Katayama, T., Ogawa, K., Ohashi, H., Kimura, H., Takahashi, S., Takeshita, K., Tomizawa, H., Goto, S., Ishikawa, T. \& Yabashi, M. (2013b). New J. Phys. 15, 083035 .

Vartanyants, I., Singer, A., Mancuso, A., Yefanov, O., Sakdinawat, A., Liu, Y., Bang, E., Williams, G. J., Cadenazzi, G., Abbey, B., Sinn, H., Attwood, D., Nugent, K. A., Weckert, E., Wang, T., Zhu, D., Wu, B., Graves, C., Scherz, A., Turner, J. J., Schlotter, W. F., Messerschmidt, M., Lüning, J., Acremann, Y., Heimann, P., Mancini, D. C., Joshi, V., Krzywinski, J., Soufli, R., Fernandez-Perea, M., Hau-Riege, S., Peele, A. G., Feng, Y., Krupin, O., Moeller, S. \& Wurth, W. (2011). Phys. Rev. Lett. 107, 144801.

Yang, J., Fan, D., Wang, S. \& Gu, Y. (2000). J. Opt. Soc. Am. A, 17, 790-793. 